\title{
Analyzing, investigating and calculating the optimum mode of the parameters affecting the record of the javelin throw including the initial velocity, initial angle, and initial height of throw
}

\author{
(D) Samaneh Mohammadia ${ }^{1}$, (D) Yousef Jalalabadi ${ }^{1}$, DFateme Nikkhoo Amiri ${ }^{2}$ \\ ${ }^{1}$ Department of Engineering, Kashmar Branch, Islamic Azad University, Kashmar, Iran. \\ ${ }^{2}$ Department of Corrective Exercise, Physical Education and Sports Sciences, University of Tehran, Tehran, Iran.
}

\begin{abstract}
The javelin throw is a track and field event, which is of very great antiquity. The most important goal in the javelin throw is the throwing record. In the javelin throw, three factors (initial velocity $V_{1}$, initial throw angle $\theta_{1}$ and initial throw height $\mathrm{H}$ ) play a fundamental role in an athlete's record. Throwing record only depends on these three factors, but of course each of them alone is subject to various factors at the time of throwing: the place, time, environmental and physical conditions of an athlete. In this study, these factors were expressed, analyzed and investigated, and the physical, technical and computational parameters with greater precision (from the perspective of sports engineering) were also expressed, and thus the optimum modes for each of them were obtained. The initial throw velocity $\left(V_{1}\right)$, initial throw angle $\left(\theta_{1}\right)$, initial throw height $(\mathrm{H})$, their roles in throwing record and the technical parameters affecting them have been investigated. Results show that the equations of optimum modes were obtained for the three aforementioned variables. Depending on a series of specific initial values for each athlete, an optimum mode of initial velocity, initial angle and initial height of throw were obtained for each athlete, resulting in the maximum record for throwing.
\end{abstract}

Keywords. Initial height, initial velocity, sports engineering, javelin throw.

\section{Introduction}

The javelin throw is a track and field event, which is of very great antiquity. In considering the records achieved in the javelin throw, a remarkable advancement with the passage of time is seen, and this record increase is not because of the increase in the people's strength at the present time compared to the past, but as a result of more precise studies and an engineering view on this movement, so that higher records are achieved by fixing the defects in the movement and using the appropriate techniques, and this process is continuous. However, the scrupulous investigation of each science and the relationship between that science and other sciences within the scope of application is called the engineering of that science. Countries, which invest in this regard (sports engineering), will make a significant progress in athletic fields and records. The current century is that of technology and the era of computer and space and as such, one cannot be oblivious to this fact and act traditionally. The technique of a projectile motion plays an important role in achieving a record (Dai et al., 2013), and in some situations, by observing a proper technique

Received: January 23, 2019 - Accepted: March 13, 2019 - Published: March 30, 2019

To cite this article: Mohammadi S, Jalalabadi Y, Amiri FN. Analyzing, investigating and calculating the optimum mode of the parameters affecting the record of the javelin throw including the initial velocity, initial angle, and initial height of throw. Turk J Kinesiol, 2019; 5(1): 1-14.

$凶$ Y. Jalalabadi, e-mail: jalalabadi2100@yahoo.comＤOI: 10.31459/turkjkin.516625 
even by athletes having greater physical strengths, can be overtaken, as experience confirms this fact. Thus there are heroes in this field who do not have extraordinary strengths, but have achieved very good records (Dai et al., 2011). In order to be able to find appropriate techniques for athletes, this sport need be observed from the engineering perspective.

\section{Methods}

Projectile motion is a form of motion in which an object (projectile) moves along a parabolic path. Generally, in the javelin throw, three factors (initial velocity $V_{1}$, initial throw angle $\theta_{1}$ and initial throw height $\mathrm{H}$ ) play a fundamental role in an athlete's record. And to achieve greater records in the javelin throw, there is a need to increase the speed of throwing, by the upper limbs, in a series of continuous and consecutive movements (Dai et al., 2013; Leigh et al., 2008; Leigh et al., 2010; Leigh et al., 2013).

Conversely, throwing record only depends on these three factors, but of course each of them alone is subject to various factors at the time of throwing: the place, time, environmental and physical conditions of an athlete (Dai et al., 2013; Leigh et al., 2008; Leigh et al., 2010). In this study, the optimum modes were achieved for athletes by calculation and from an engineering perspective (depending on the place, time, environmental and physical conditions of athletes), and were placed at their disposal to achieve the maximum possible record; which in their capacity, depend on the place, time and circumstances. As shown in Figure 1, not only does each of the effective parameters depend on some factors, but they also have relationships with each other, and thus obtaining the optimum mode of one of the parameters may cause a decrease in some other parameters and consequently cause reduction of record, therefore, the optimum mode of all three parameters should be obtained simultaneously. For this purpose, first, the optimum modes of each parameter were investigated, and in the end, intersection points of these parameters were located, by drawing diagrams.

In a research on the relationship between the diversity of techniques and the performance presented by the elite javelin throwers during the match, a negative correlation was observed between the diversity of technique and the performance in men throwers, but it was not the case in women throwers. Thus, the more uniform movement pattern is used by men in throwing, the better records they achieve, depends on the throw angle, the arm length, the initial throw velocity and in general, their techniques (Dai et al., 2013).

\section{Procedure}

To obtain the formula for the range of a projectile (record), there are two methods used, one of which has been proven:

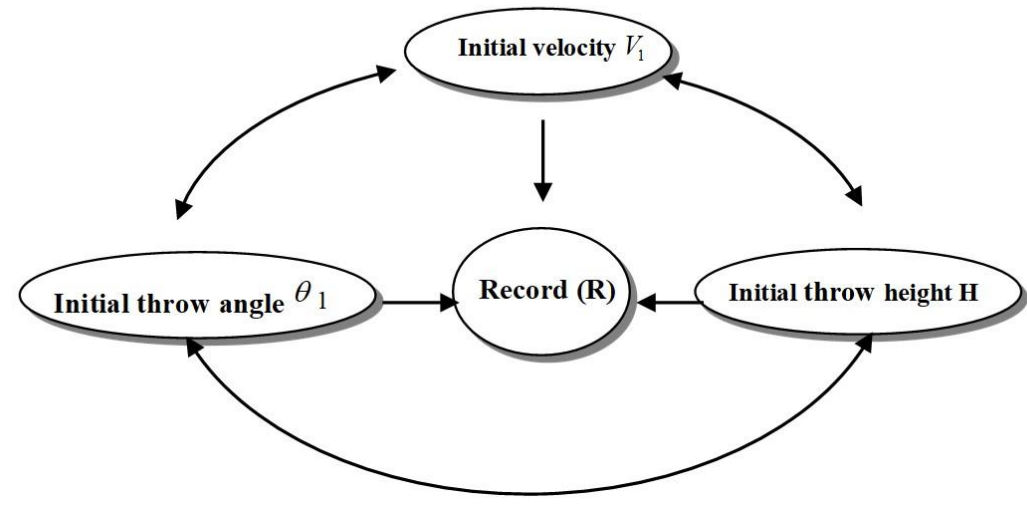

Figure 1. Effective parameters depend on some factors. 
In this method, the equations of projectile motion are used and are shown as follows:

$$
\begin{aligned}
& y=-\frac{1}{2} g t^{2}+V_{1 y} t+y_{0} \quad, \quad V_{1 y}=V_{1} \sin \left(\theta_{1}\right) \quad, \quad y=-H \quad, \quad y_{0}=0 \Rightarrow \\
& \Rightarrow \quad-H=-\frac{1}{2} g t^{2}+V_{1} \sin \left(\theta_{1}\right) t+0 \quad \Rightarrow \\
& \Rightarrow+\frac{1}{2} g t^{2}-V_{1} \sin \left(\theta_{1}\right) t-H=0 \quad \Rightarrow \\
& \Rightarrow \quad t=\frac{V_{1} \sin \left(\theta_{1}\right) \pm \sqrt{V^{2}{ }_{1} \sin ^{2}\left(\theta_{1}\right)+2 g H}}{g} \quad, \quad R=V_{1 x} t \\
& \Rightarrow \quad R=V_{1} \cos \left(\theta_{1}\right) \frac{V_{1} \sin \left(\theta_{1}\right) \pm \sqrt{V^{2} \sin ^{2}\left(\theta_{1}\right)+2 g H}}{g} \Rightarrow \\
& \Rightarrow \quad R=\frac{V^{2}{ }_{1} \cos \left(\theta_{1}\right) \sin \left(\theta_{1}\right) \pm V_{1} \cos \left(\theta_{1}\right) \sqrt{V^{2}{ }_{1} \sin ^{2}\left(\theta_{1}\right)+2 g H}}{g} \\
& R=\frac{V_{1} \cos \left(\theta_{1}\right)}{g}\left(V_{1} \sin \left(\theta_{1}\right)+\sqrt{V_{1}^{2} \sin ^{2}\left(\theta_{1}\right)+2 g H}\right)
\end{aligned}
$$

According to the final equation (Equation 1), it can be seen that the range of a projectile is directly related to the initial velocity $V_{1}$, initial throw angle $\theta_{1}$, and initial throw height $\mathrm{H}$. Thus, each of them plays a role in the final range to some percent, among which the initial velocity $V_{1}$ is of greater importance than the rest of them.

As shown in Equation 1, the range of a projectile is directly related to the initial velocity $V_{1}$, initial throw angle $\theta_{1}$, and initial throw height $\mathrm{H}$. Moreover, each of them plays a role in the final range to some percent, among which the initial velocity $V_{1}$ is of greater importance than the rest of them (diagrams 1, 2 and 3); the initial throw velocity in projectile motion depends on the following two factors:

A) The velocity vector of the center of mass of the athlete's body $\boldsymbol{u}_{1}$

B) The velocity vector of the athlete's wrist in relation to their body (from the view of an observer on the person's body) $u_{2}$

As shown in Figure 3, the resultant of these two velocity vectors is the ultimate velocity vector, and it determines the initial throw velocity.

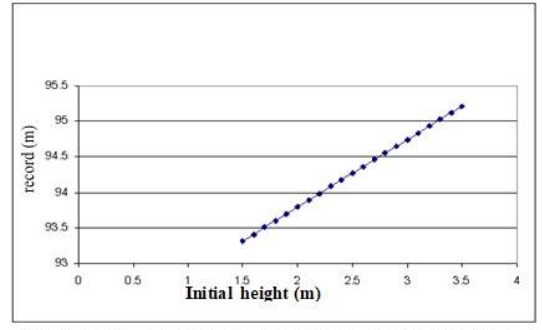

Diagram.3: Chart records changes in elevation than the initial throw (Initial speed $=30 \mathrm{~m} / \mathrm{s} \&$ initial angl $=45^{*}$ )

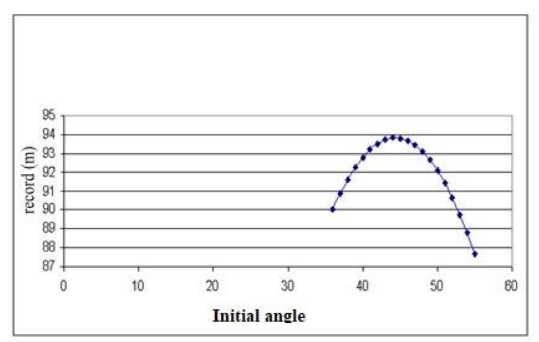

Diagram.2: Chart records throw with the initial throw angle (Initial speed $=30 \mathrm{~m} / \mathrm{s}$ \& initial height=2m)

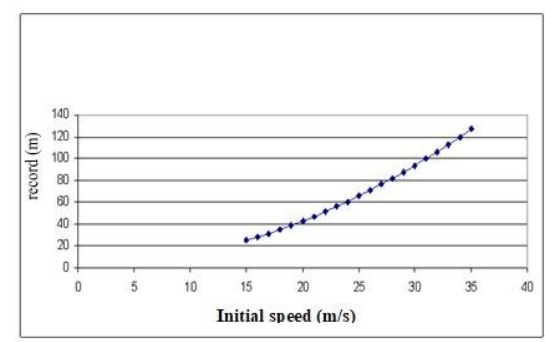

Diagram.1: Chart records throw with the initial speed throw (Initial throw $=45$ \& initial height $=2 \mathrm{~m}$ ) 


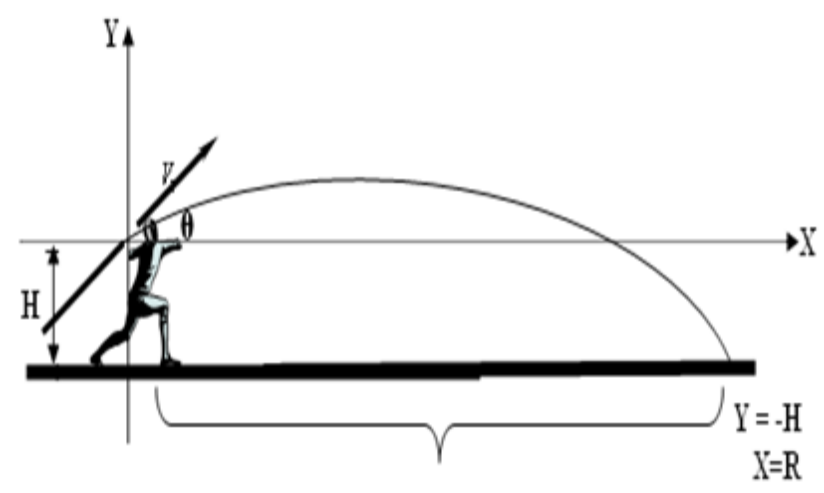

Figure 2. The record of javelin throws.

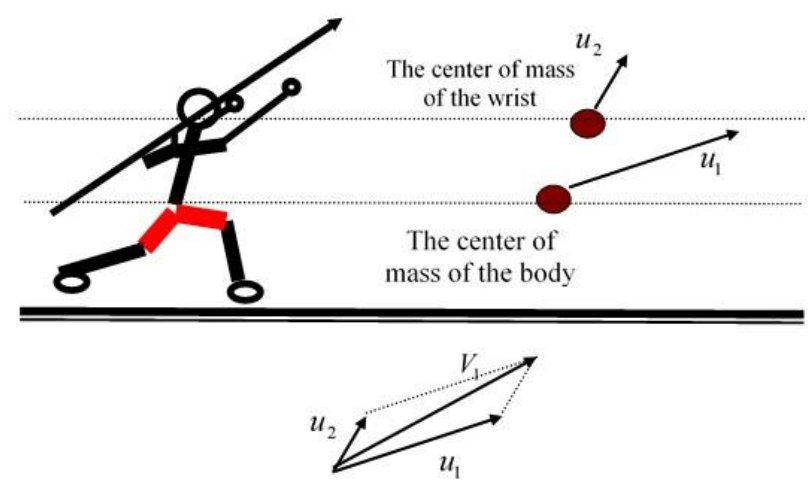

Figure (3): The resultant of the two velocity vectors using the parallelogram method

The resultant of these two vectors can be calculated using two methods: 1) trigonometric and 2) Cartesian.

A) The trigonometric method: In this method, the resultant is obtained from the relationship Equation 2 and Figure 4, and the result depends on the velocities $\boldsymbol{u}_{1}$ and $\boldsymbol{u}_{2}$ and the angle between these two $(\theta)$.

$$
V_{1}=\sqrt{u_{1}^{2}+u_{2}^{2}+2 u_{1} u_{2} \cos (\theta)}
$$

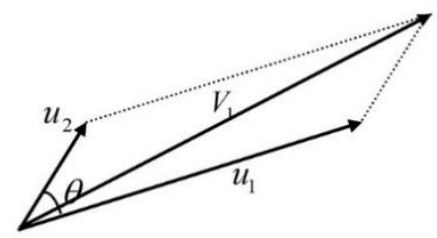

Figure 4. The resultant of two vectors in polar coordinates.
B) The Cartesian method: In this method, the velocity vectors $\boldsymbol{u}_{1}$ and $\boldsymbol{u}_{2}$ are converted into vectors in the Cartesian coordinate system and these two vectors are then added.

As shown in Equation 2 the value of the initial velocity $V_{1}$ depends on the values of the velocities $u_{1}$ and $u_{2}$ and the angle between them $\theta$. However, the greater the values of the velocities $u_{1}$ and $u_{2}$ are and the less the angle $\theta$ is, the greater the value of the velocity $V_{1}$ will be, and subsequently the greater the record of the projectile.

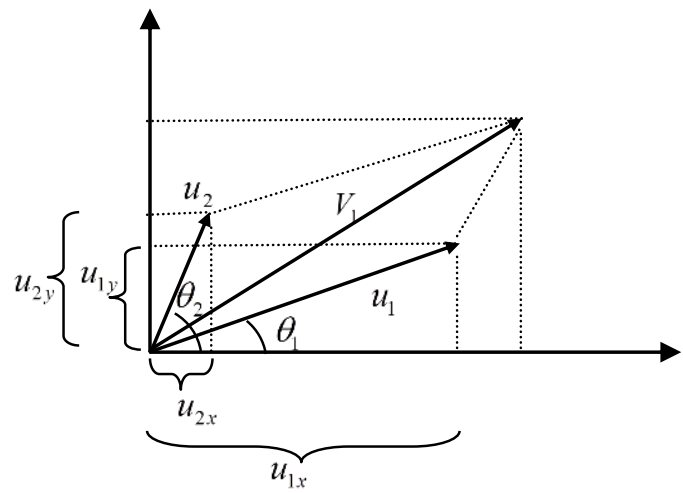

Figure 5. The resultant of two vectors in Cartesian coordinates.

$$
\begin{array}{ll}
V_{1}=\sqrt{V_{x}^{2}+V_{y}^{2}} & \\
V_{x}=u_{1 x}+u_{2 x} & , \quad V_{y}=u_{1 y}+u_{2 y} \\
u_{1 x}=u_{1} \cos \left(\theta_{1}\right) & , \quad u_{2 x}=u_{2} \cos \left(\theta_{2}\right) \\
u_{1 y}=u_{1} \sin \left(\theta_{1}\right) & , \quad u_{2 y}=u_{2} \sin \left(\theta_{2}\right)
\end{array}
$$

Based on Equation 3, it can be seen that the value of the initial velocity $V_{1}$ depends on the values of the velocities $u_{1}$ and $u_{2}$, and the angles $\theta_{1}$ and $\theta_{2}$. And each of these two factors (the velocities $u_{1}$ and $u_{2}$ ) depends on some parameters as well. According to Figure (5) we can write: $\theta=\theta_{2}-\theta_{1}$

Based on Equation 3, it can be concluded that the velocity $V_{1}$ depends on the values of the velocities $u_{1}$ and $u_{2}$, the angles $\theta_{1}$ and $\theta_{2}$, and generally depends on the velocity vectors $\vec{u}_{1}$ and $\vec{u}_{2}$. Here, each of these parameters was examined separately. 
Velocity vector $\vec{u}_{1}$ (the velocity of an athlete's center of mass at throw)

The velocity of an athlete's center of mass at throw is a combination of two velocities: horizontal velocity $u_{1 x}$ and vertical velocity $u_{1 y}$ (Figure 6), that each one, in turn, are dependent on some factors.

$$
u_{1}=\sqrt{u_{1 x}^{2}+u_{1 y}^{2}}
$$

Based on the above relation (Equation 5), as athlete increase the horizontal velocity $u_{1 x}$ and vertical velocity $u_{1 y}$, the velocity $\boldsymbol{u}_{\mathbf{1}}$ increases as well, which is also directly related to the velocity $v_{1}$, and by increasing $V_{1}$, the record of projectile motion can be improved. But there are limits on these velocity increases, and they cannot be increased up to their maximum capacity all at the same time. Thus, this is because the increase in all subcategory parameters at the same time in some cases, results in a decrease in the main parameters, and this factor causes a decrease in the record, as such, the parameters need to be analyzed, and an optimum mode to be discovered. First, the analyzes each subcategory parameters were dealt with, and in the end we analyze the parameters of higher categories.

The horizontal velocity $u_{1 x}$ is an athlete's forward velocity, and this parameter depends on the athlete's ability and technique. The higher the athlete's velocity and strength during sprints (Figure 7), the higher horizontal velocity $u_{1 x}$ they can produce, but the limitation here is that, when throwing javelin with proper angle $\theta_{1}$, which plays a significant role in the throw and the projectile record, the athlete cannot perform the throwing action under these conditions. If an athlete wants to use their maximum horizontal velocity in a throw, they will no longer be able to move their body in the direction of the y-axis by changing the center of mass of their body, while on the move (which is impossible) to cause an upward movement. Therefore, the more athletes would want to increase their upward velocity, the more they would have to reduce their maximum horizontal velocity, and as such, an optimum mode for the athlete is looked for in this study (This optimum mode can be found by calculation, and performing a series of tests on the athlete, according to the software program).

As shown in Figure 6, it can be observed that, if athletes uses the maximum capacity of their horizontal velocity, the initial throw angle $\left(\theta_{1}\right)$ will decrease, and this results in a decrease in the record; and even if athletes, by moving their hand while sprinting, wants to increase the angle $\theta_{2}$ or the velocity $u_{2}$ in order to increase the velocity, then they would have to reduce the angle $\theta_{2}$, and in order to increase the angle $\theta_{2}$, they would have to reduce the velocity $u_{2}$. Further, in order to increase the angle $\theta_{2}$ without changing the velocity $u_{2}$, they would have to reduce the $u_{1 x}$ and vice versa. Thus, according to the tests carried out, the velocity $\boldsymbol{u}_{2}$ is inversely related to the $u_{1 x}$ and $\theta_{2}$. Moreover, the mode in Figure 6-b is not the optimum mode for throwing. By considering the above analyses, it can be concluded that the center of mass of an athlete's body should have an upward move, and the athlete performs this action by bending their body backward into the shape of an arch. This body opening and changes in the center of mass of the body in the vertical direction takes place at the last step, and raises the center of mass with an upward velocity in the vertical direction relative to the body of the athlete (Bosen, 1985; Robinson, 2016). This action of the athlete will contribute tremendously to the velocity and appropriate throw angle. By this action, the initial release height of the javelin also increases. The vertical velocity $u_{1 y}$ is an athlete's upward velocity, and this parameter depends on the athlete's ability and technique. In this study, based on earlier analysis, a theoretical and practical conclusion is drawn that the optimum mode for a throw will occur if the center of mass of the body has its movement and velocity in the vertical direction, thus this action helps in obtaining the best optimum angle of throw, initial height of throw, and balance after throwing. In addition, the velocity in the direction perpendicular 
to the center of mass of the body has an optimum mode, which depends on other parameters. In order to be able to produce movement and velocity for the body in vertical direction, athletes should fold their body and then open it in vertical direction. Depending on the degree of velocity that athletes produces for their body in the vertical direction, they have to reduce their maximum horizontal velocity by that degree, and therefore this study seek an optimum mode for the intended athlete, based on the abilities and conditions that exist.

In general, the greater the horizontal velocity $u_{1 x}$ and vertical velocity $u_{1 y}$ athletes have, the better record they can achieved by observing the correct technique, and these parameters only depend on the athlete's physical, emotional and psychological characteristics. For research and analysis in this case, we enter non-biomechanical sciences, which should be entrusted to the experts in their fields of specialty (Diagram 1).

Velocity vector $\vec{u}_{2}$ : (The velocity of an athlete's wrist at throw)

The velocity of the athlete's wrist, in which the javelin is placed, is produced by changing the center of mass of the hand (the set of hand, forearm and arm) by the athlete, which depends on the following factors according to the theoretical and practical studies conducted:

\section{1) The athlete's physical strength and physical fitness:}

The higher the athlete's physical strength and physical fitness and the more athletics upper limbs are, the greater the velocity $u_{2}$ he can produce.

2) The athlete's body type (the size of the palm and fingers, the length of the forearm and arm):

The size of the wrist and palm contributes tremendously to an athlete with regard to the balance (a proper and optimum throw angle), and the release velocity of the javelin, and these factors increase the record of a throw. The greater the contact area between the javelin and the hand, the better the transference of force, which takes place through the hand, and the easier the maintenance of balance of the javelin by the athlete (Bosen, 1985; Bayat et al., 2015). Some minor changes to some extent, in taking the javelin by hand, can cause an optimum mode to be achieved. The length of an athletic forearm and arm, plays an important role in the velocity $\boldsymbol{u}_{2}$, the longer the athletic forearm and arm are, the greater the torque generated around the axis of rotation in which the shoulder is attached, causing an increase in the $u_{2}$ (David et al., 2018).

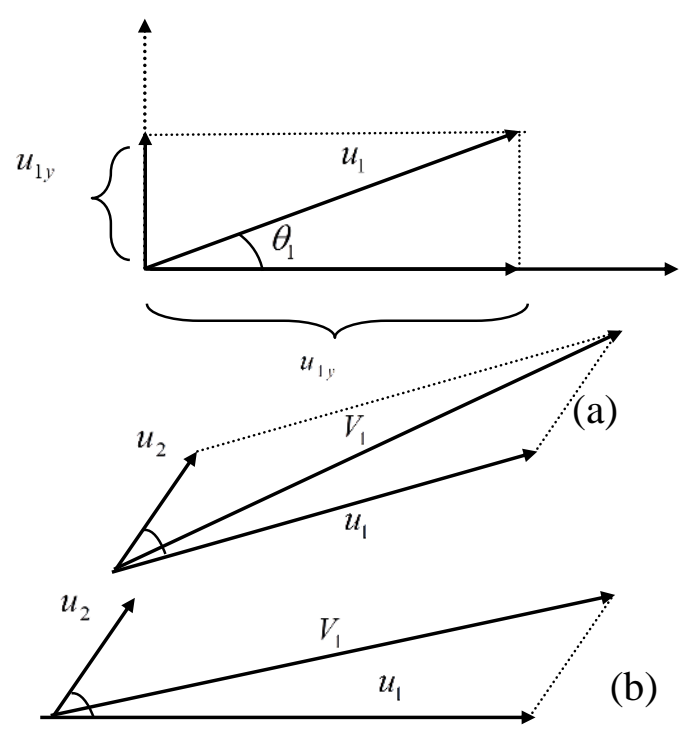

Figure 6. a) The correct mode of velocities, b) The incorrect mode of velocities.

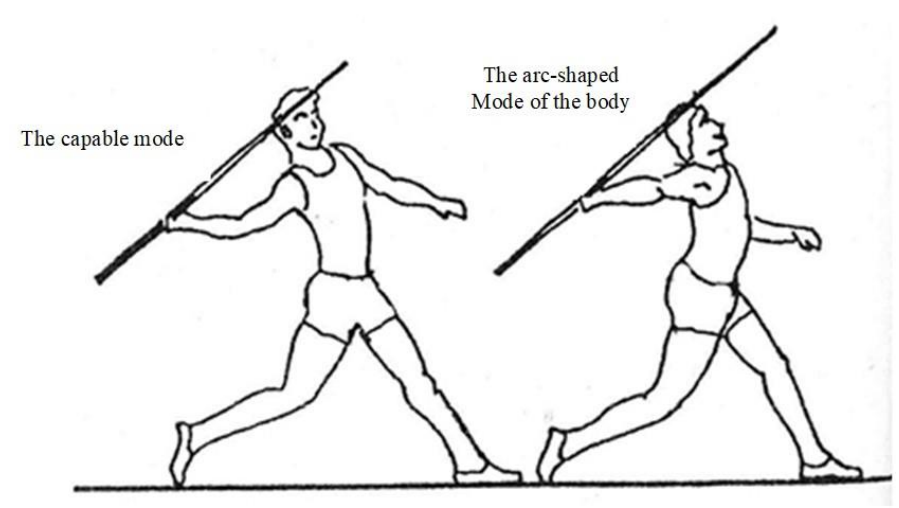

Figure 7. Throwing techniques. 


\section{3) The velocity $u_{1}$ :}

The maximum velocity $u_{2}$ that an athlete can build, is in a mode that $u_{1}=0$, and as the velocity $u_{1}$ increases, the athlete can practically build a lower velocity $u_{2}$. Thus, the velocity $u_{2}$ is inversely related to the velocity $u_{1}$. Here, since this study seeks to achieve and build the maximum velocities $u_{2}$ and $u_{1}$ , therefore, an optimum mode is looked for, and this optimum mode can be implemented using several methods. As shown in Figure 8-b, the optimum mode $u_{2}$ is a combination of the velocities in the direction of $\mathrm{x}$ and $\mathrm{y}$ axes. In Figure $8-\mathrm{c}, u_{2}$ is completely in line with the y-axis, and in Figure 8-d, $u_{2}$ is completely in line with the $x$-axis, so that the former reduces the value of the initial velocity $V_{1}$, and the latter reduces the initial throw angle $\left(\theta_{1}\right)$, hence in both cases, a decrease in the record is observed (Best et al., 1993; Bartlett \& Best, 1988).
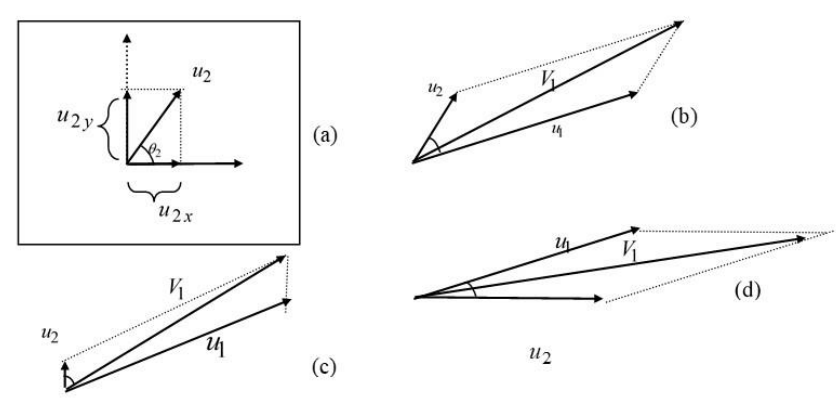

Figure 8. a) The velocity $u_{2}$ and its components, b) The optimum mode of the velocity $u_{2}, \mathrm{c}$ ) The mode in which the velocity $u_{2}$ is completely in line with the y-axis, $\mathrm{d}$ ) The mode in which the velocity $u_{2}$ is completely in line with the $x$-axis.

\section{The initial throw angle $\theta_{1}$ :}

In projectile motion, one of the parameters which plays an important role in the range of the projectile (the record) is the initial throw angle (Liu et al., 2010a; Leigh et al., 2010). However, When the initial throw angle is 90 degrees, the motion is called the projectile motion in vertical direction, and in this type of projectile motion, the record, which is the displacement, in line with the $\mathrm{x}$-axis, is equal to zero. Thus there is no displacement in the direction of motion, and as the angle decreases from 90 degrees, the range of motion increases, and the maximum range at a certain angle is ascertained, and if the throw angle is reduced, a decrease in the range of the projectile is seen (Diagram 2 and Figure 9). In a projectile motion in which the throw point along with the landing point are on the horizontal axis at the ground level and the throw points be considered the origin of the coordinate system, the equation of the range of the projectile motion is obtained from the following relation, and according to this equation, the maximum range is obtained at a $45-$ degree angle to the horizon:

$$
\text { Range of motion } R=\frac{V_{1}^{2} \sin (2 \theta)}{2 g}
$$

In order to obtain the angle of maximum range, the range of equation has to be differentiated with respect to the angle, to find its extreme point, which is as follows (1):

$$
\begin{aligned}
& \frac{d}{d \theta}(R)=\frac{d}{d \theta}\left(\frac{V_{1}^{2} \sin (2 \theta)}{2 g}\right)=\frac{V_{1}^{2}}{2 g} \frac{d}{d \theta}(\sin (2 \theta) \\
& =\frac{V_{1}^{2}}{2 g}(2 \cos (2 \theta))=\frac{V_{1}^{2} \cos (2 \theta)}{g} \\
& \frac{d}{d \theta}(R)=0 \Rightarrow \frac{V_{1}^{2} \cos (2 \theta)}{g}=0 \\
& \Rightarrow \cos (2 \theta)=0 \Rightarrow 2 \theta=\frac{\pi}{2} \\
& \Rightarrow \theta=\frac{\pi}{4}=45^{\circ}
\end{aligned}
$$

In projectile motions in which the throw point and the landing point have a difference in height $\mathrm{H}$ and are not at the same level, there are two modes; either the throw point is higher than the landing point or vice versa (the throw point is lower than the landing point) (Figure 10). 


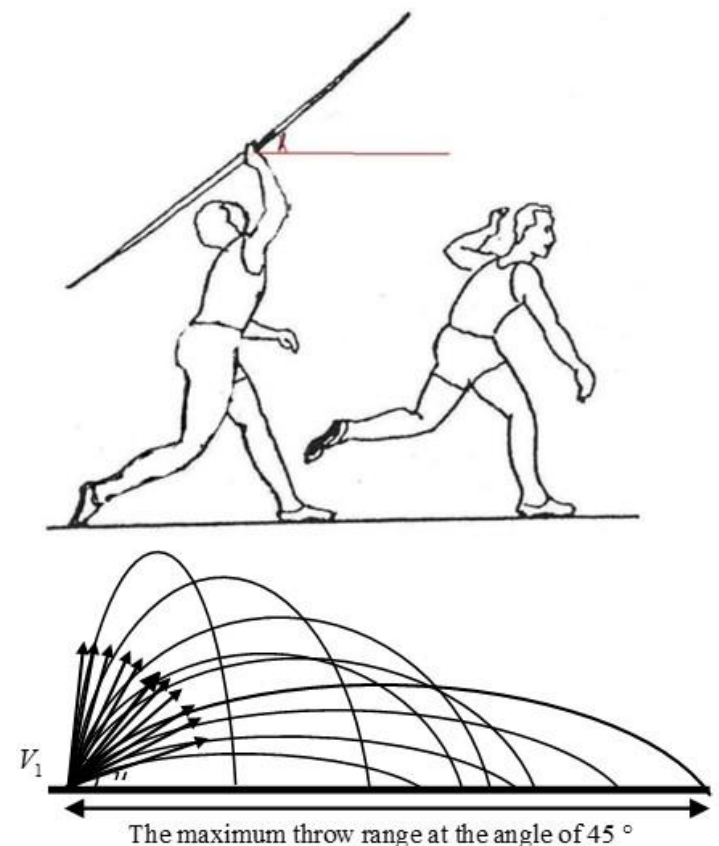

The maximum throw range at the angle of $45^{\circ}$

Figure 9. The maximum throw range.

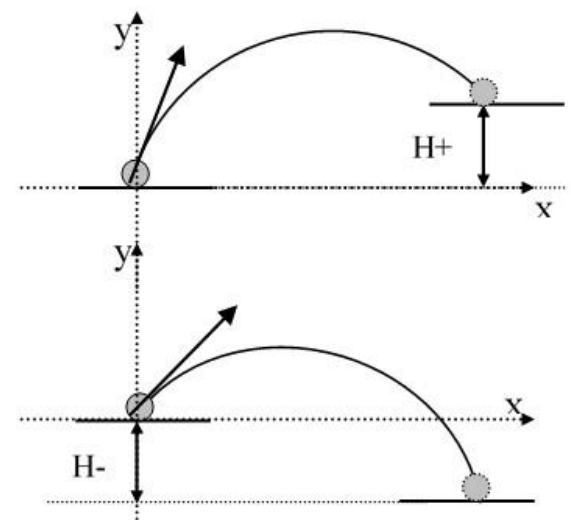

Figure 10. Two modes, either the throw point is higher than the landing point or vice versa.

In these modes, the starting point of the throw is always considered to be on the origin of the coordinate system, thus in the mode in which the throw point is higher than the landing point, the height of landing point is - $\mathrm{H}$, and in the mode that the throw point is lower than the landing point, the height of landing point is $+\mathrm{H}$. The range of motion is obtained from the In the above relation, $x$ is a displacement in horizontal direction (the range of a motion) and $\mathrm{y}$ is the positional coordinates of a landing point.
Following relation, which is known as trajectory equation:

$$
y=\frac{-g x^{2}}{2 V^{2} \cos ^{2}\left(\theta_{1}\right)}+x \tan \left(\theta_{1}\right)
$$

The record of a projectile is then given as:

$$
R=\frac{V_{1} \cos \left(\theta_{1}\right)}{g}\left(V_{1} \sin \left(\theta_{1}\right)+\sqrt{V_{1}^{2} \sin ^{2}\left(\theta_{1}\right)+2 g H}\right)
$$

Here, in order to find an appropriate angle for the maximum range, the Equation (9) has to be differentiated with respect to $\theta_{1}$, which is as follows:

$$
\frac{d}{d \theta_{1}}(R)=\frac{d}{d \theta_{1}}\left[\left(\frac{V_{1} \cos \left(\theta_{1}\right)}{g}\left(V_{1} \sin \left(\theta_{1}\right)+\sqrt{V_{1}^{2} \sin ^{2}\left(\theta_{1}\right)+2 g H}\right)\right]\right.
$$

After performing complete differentiation, the following relation will be achieved:

$$
\begin{aligned}
& \frac{d}{d \theta_{1}}(R)=\frac{d}{d \theta_{1}}\left[\left(\frac{V_{1} \cos \left(\theta_{1}\right)}{g}\left(V_{1} \sin \left(\theta_{1}\right)+\sqrt{V_{1}^{2} \sin ^{2}\left(\theta_{1}\right)+2 g H}\right)\right]\right. \\
& \theta_{1}=\sin ^{-1}\left(\sqrt{\frac{A}{A^{2}+2 A}}\right) \\
& A=\frac{2 g H}{V_{1}^{2}}
\end{aligned}
$$

It can be seen in the obtained formula that the optimum angle for the throw depends on factors such as the initial throw velocity $\left(V_{1}\right)$ and initial throw height $(\mathrm{H})$.

However, in Table 1, the optimal values of the initial throw angle are calculated for different values of initial throw velocity $\left(V_{1}\right)$ and initial height $(\mathrm{H})$.

Furthermore, the results of previous studies conducted show a close relationship between the parameters of motion, so that each one, in some way, is dependent on other parameters, and this relationship is direct and/or inverse, and the optimum mode can be determine using completely accurate methods (Sports Engineering), and should be put at the athlete's disposal, and the exercises of the next stage and/or a match should be performed based on the existing parameters (Figure 11). 


\section{Table 1}

The optimal values of the initial throw angle are calculated for different values of initial throw velocity (v1) and initial height $(\mathrm{H})$.

\begin{tabular}{cccccc}
\hline $\begin{array}{c}\text { Initial Height } \\
\text { of Throw }(\mathrm{m})\end{array}$ & $\begin{array}{c}\text { Optimal Angle } \\
\text { for Velocity } 15 \\
\mathrm{~m} / \mathrm{s}\end{array}$ & $\begin{array}{c}\text { Optimal Angle } \\
\text { for Velocity } 20 \\
\mathrm{~m} / \mathrm{s}\end{array}$ & $\begin{array}{c}\text { Optimal Angle } \\
\text { for Velocity } 25 \\
\mathrm{~m} / \mathrm{s}\end{array}$ & $\begin{array}{c}\text { Optimal Angle } \\
\text { for Velocity } 30 \\
\mathrm{~m} / \mathrm{s}\end{array}$ & $\begin{array}{c}\text { Optimal Angle } \\
\text { for Velocity } 35 \\
\mathrm{~m} / \mathrm{s}\end{array}$ \\
\hline 1 & 43.80 & 44.31 & 44.55 & 44.69 & 44.77 \\
1.1 & 43.68 & 44.24 & 44.51 & 44.66 & 44.75 \\
1.2 & 43.57 & 44.18 & 44.47 & 44.63 & 44.72 \\
1.3 & 43.46 & 44.11 & 44.42 & 44.60 & 44.70 \\
1.4 & 43.35 & 44.04 & 44.38 & 44.56 & 44.68 \\
1.5 & 43.24 & 43.98 & 44.34 & 44.53 & 44.66 \\
1.6 & 43.13 & 43.91 & 44.29 & 44.50 & 44.63 \\
1.7 & 43.02 & 43.85 & 44.25 & 44.47 & 44.61 \\
1.8 & 42.91 & 43.78 & 44.21 & 44.44 & 44.59 \\
1.9 & 42.80 & 43.72 & 44.17 & 44.41 & 44.57 \\
2 & 42.70 & 43.66 & 44.12 & 44.38 & 44.54 \\
2.1 & 42.59 & 43.59 & 44.08 & 44.35 & 44.52 \\
2.2 & 42.49 & 43.53 & 44.04 & 44.32 & 44.50 \\
2.3 & 42.38 & 43.47 & 44.00 & 44.29 & 44.48 \\
2.4 & 42.28 & 43.40 & 43.96 & 44.27 & 44.46 \\
2.5 & 42.18 & 43.34 & 43.91 & 44.24 & 44.43 \\
2.6 & 42.08 & 43.28 & 43.87 & 44.21 & 44.41 \\
2.7 & 41.97 & 43.22 & 43.83 & 44.18 & 44.39 \\
2.8 & 41.87 & 43.15 & 43.79 & 44.15 & 44.37 \\
2.9 & 41.78 & 43.09 & 43.75 & 44.12 & 44.35 \\
3 & 41.68 & 43.03 & 43.71 & 44.09 & 44.32 \\
\hline
\end{tabular}

Initial throw height $H$ :

As shown in Equation 1, the initial throw height is also directly related to the range of javelin motion, and the more the initial throw point is increased, the more the throw record will also increase (Figure 3). In fact, it is of less importance compared to the initial throw velocity $\left(V_{1}\right)$ and the initial throw angle $\theta_{1}$, but in conditions of close competition among athletes, observing the technical hints in adjusting the throw point and applying the initial throw height in the optimum mode of the two aforesaid parameters ( $V_{1}$ the initial throw velocity and $\theta_{1}$ the initial throw angle) can help a great deal to achieve a better position:

$$
R=\frac{V_{1} \cos \left(\theta_{1}\right)}{g}\left(V_{1} \sin \left(\theta_{1}\right)+\sqrt{V_{1}^{2} \sin ^{2}\left(\theta_{1}\right)+2 g H}\right)
$$

The Initial throw height depends on the following factors:

1) The athlete's body type, 2) The athlete's technique,

3) The initial throw velocity $V_{1}, 4$ ) The initial throw angle $\theta_{1}$

\section{The athlete's body type}

One of the most important characteristics that a javelin throwing athlete must possess in order to achieve a higher record ( $R$ ) is an appropriate body type. For an athlete, the body type includes the following items:

\section{A) The athlete's height B) Physical, emotional and mental fitness}




\section{A) The athlete's height}

The athlete's height is also directly related to the initial height $(\mathrm{H})$ of the throw point (Diagram 3). According to the relation below, the higher the height of the initial throw, the greater the record (R) of a projectile. Also, the greater the increase in the release height of the javelin, the greater the horizontal range of the javelin and this is shown in the relation below:

The record of a projectile is given as:

For example, two athletes, which are assumed to have projectiles with identical initial velocities and throw angles, but with different initial throw heights, their records under ideal conditions according to Equation (11), are as follows:

The athlete number 1: $\left\{\begin{array}{l}V_{1}=25 \mathrm{~m} / \mathrm{s} \\ \theta_{1}=37 \\ H_{1}=2 \mathrm{~m}\end{array}\right\} \Rightarrow R_{1}=62.58 \mathrm{~m}$

The athlete number 2: $\left\{\begin{array}{l}V_{1}=25 \mathrm{~m} / \mathrm{s} \\ \theta_{1}=37 \\ H_{1}=1.8 \mathrm{~m}\end{array}\right\} \Rightarrow R_{2}=62.31 \mathrm{~m}$

Based on the calculations performed, it can be seen that a difference in throw height of $20 \mathrm{~cm}$, results in a difference in record of $27 \mathrm{~cm}$. Thus it can be concluded that the higher the throw height, the better the athlete's record, and one of the factors influencing hugely the throw height, is the athlete's height and body type. In fact, this point must not be forgotten that the throw height can be adjusted to some extent by the athlete and this adjustment has a minimum and maximum point. Consequently, these changes in the throw height may result to changes in controlling the throw angle and initial throw velocity, and by proper management and correct planning in different circumstances for athletes with different body types, the optimum modes can be obtained by calculation and experiments. An athlete's height consists of the length of forearm, the length of arm, the length of back (upper body), the length of thigh and the length of shank.
The length of an athlete's forearm, arm, back (upper body), thigh and shank is directly related to the height of the throw point. Therefore, since the throw height is directly related to the record, the length of an athlete's forearm, arm, back (upper body), thigh and shank is also directly related to the record of a projectile.

An athlete's height consists of the length of these segments: forearm, arm, spine, thigh and shank.

The length of the aforementioned segments is directly related to the height of the throw point.

In addition, the length of the segments influencing the initial throw height is directly related to the horizontal range of javelin throw, and the length of these segments plays an important role in helping the initial throw velocity, however, it should be noted that the greater the length of these segments, the greater force they will generate.

\section{The athlete's technique}

An athlete, by using techniques and skills obtained from the implementation of the programs necessary to achieve a maximum throw, can achieve an appropriate throw height which depends on the aforementioned factors.

The javelin throw technique comprises of 4 phases: An approach, a 5-step rhythm, throw (throw is a part of 5-step rhythm) and recovery.

Basically, an athlete, by correctly observing the techniques of each phase, can increase the initial throw height at the release of javelin (Liu, Leigh, \& Yu, 2010a).

\section{The initial throw velocity $\left(V_{1}\right)$ :}

The lower the initial throw velocity, the better an athlete can adjust and implement the throw height. However, several studies and practical experiments carried out on athletes showed that as the velocity $u_{1 x}$ increases, the initial throw height decreases with an appropriate control. In addition, an athlete, in stopping mode $u_{1 x}=0$, will have the best control on the optimum initial throw height, but as mentioned earlier in this study, this action, on the other hand, 
reduces the initial throw velocity $V_{1}$ and consequently reduces the record. Therefore, based on the relationship between these parameters, the optimum mode can be found.

\section{Initial throw angle $\left(\theta_{1}\right)$}

The initial throw angle is also proportional to the initial throw height (but of less importance), as the initial throw angle increases, the initial throw height increases as well, but as mentioned earlier in this study that beyond a certain angle, a decrease in the record is observed (Liu, Leigh, \& Yu, 2010b). Therefore, in this situation also, an optimum mode, depending on the existing parameters should be determined.

\section{Results}

In this study, the changes in the range of motion (the record) and the variables affecting it are calculated and obtained.

The relationship between the range of motion (the record) and the initial velocity is shown in Diagram 1.

The relationship between the range of motion (the record) and the initial throw angle is shown in Diagram 2 on the assumption that the two other parameters are fixed.
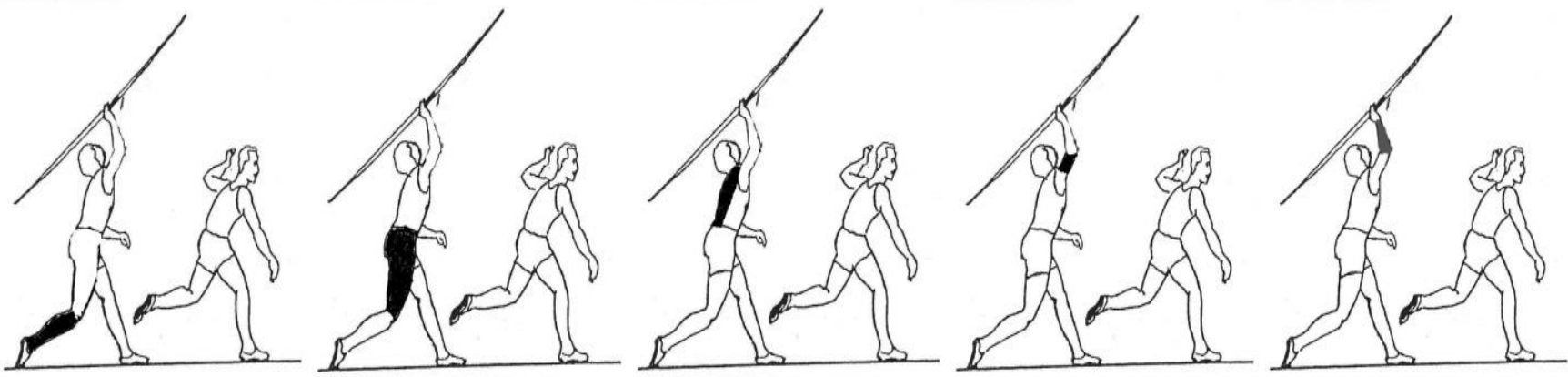

Figure 11. An athlete's height consists of the length of forearm, the length of arm, the length of back (upper body), the length of thigh and the length of shank.

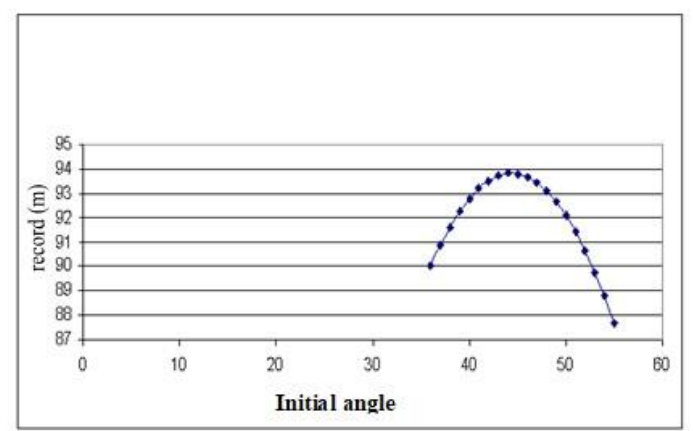

Diagram.2: Chart records throw with the initial throw angle (Initial speed $=30 \mathrm{~m} / \mathrm{s} \&$ initial height $=2 \mathrm{~m}$ )

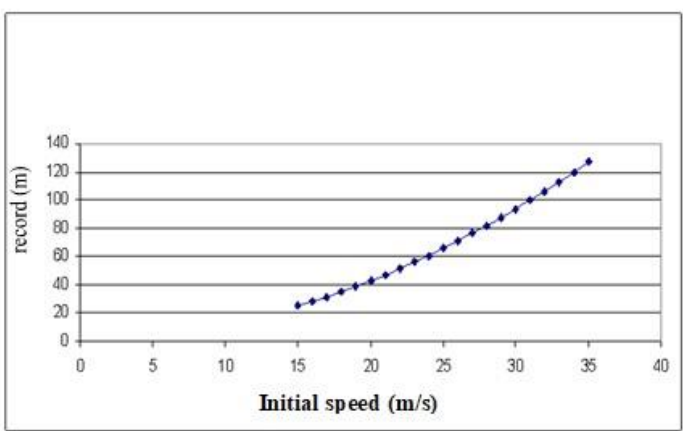

Diagram.1: Chart records throw with the initial speed throw (Initial throw $=45 \&$ initial height $=2 \mathrm{~m}$ ) 


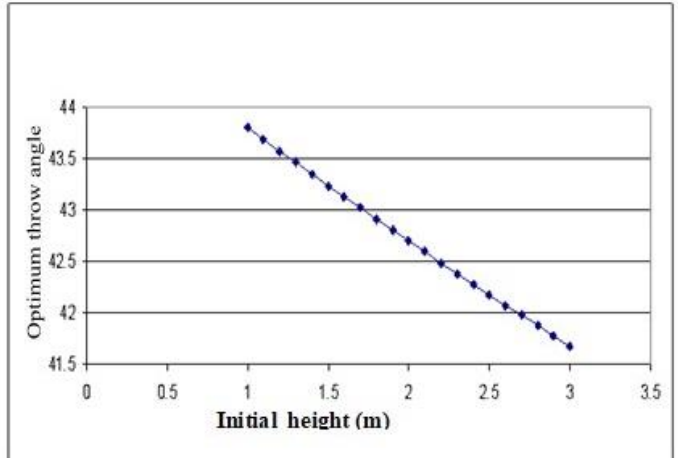

Diagram.4: Chart Optimum throw angle with height of the throw (Initial speed $=15 \mathrm{~m} / \mathrm{s}$ )

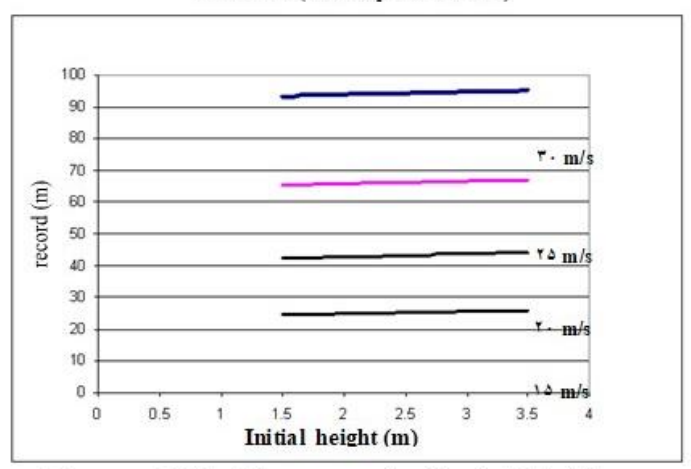

Diagram.6: Chart throws record with a height of throw in different speeds (Initial angle $=45$ )

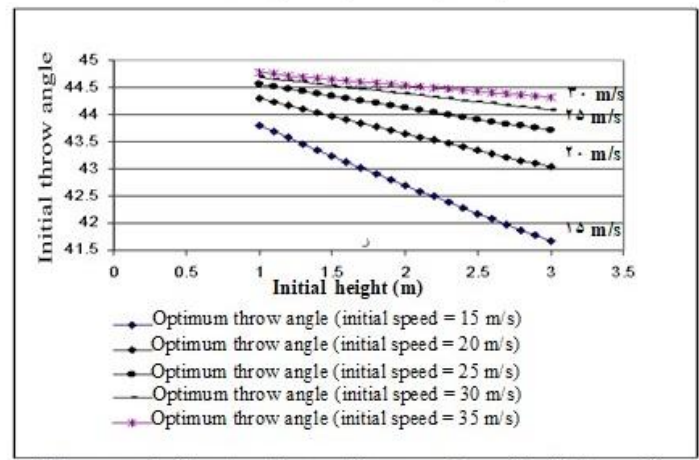

Diagram.8: Chart optimum throw angle and initial speed throw in different speeds

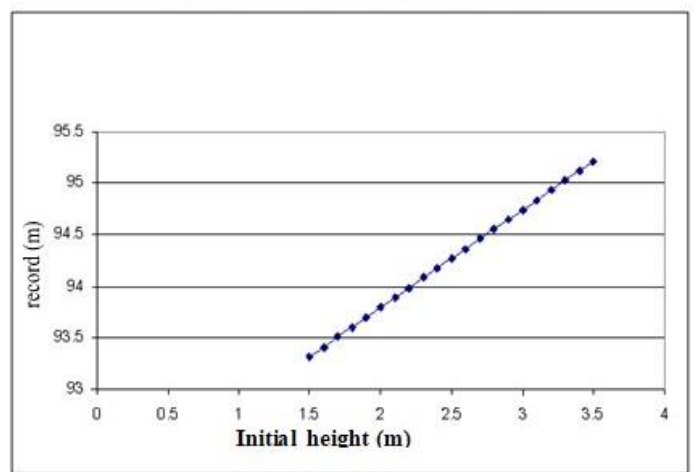

Diagram.3: Chart records changes in elevation than the initial throw (Initial speed $=30 \mathrm{~m} / \mathrm{s}$ \& initial angl $=45^{\circ}$ )

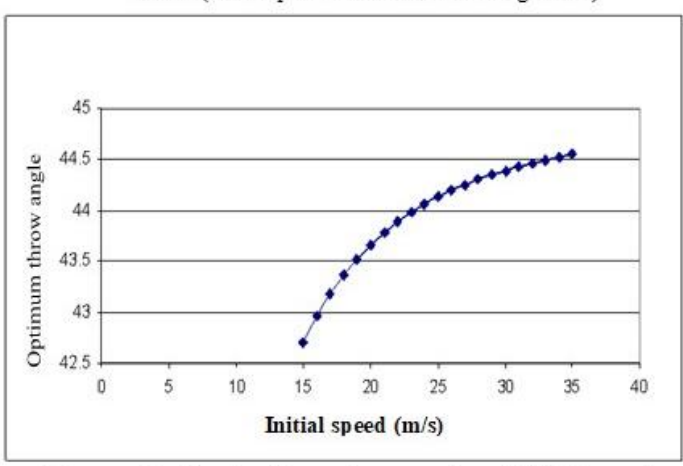

Diagram.5: Chart optimum throw angle and initial speed throw

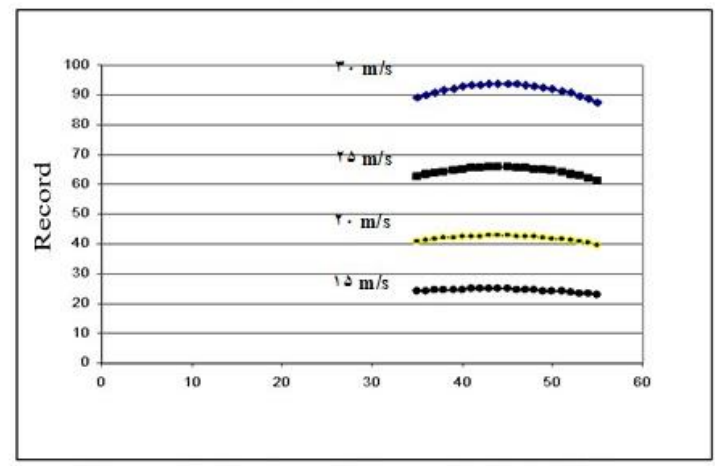

Diagram. 7: Chart records throw with the initial throw angle in different speeds

The relationships between the record and different velocities and heights are shown in Diagrams 6 and 7.

The optimum angles in different velocities and heights are shown in Diagram 8.

\section{Discussion}

The results of this study show that all three parameters are continuously related to each other, as well as the record. 


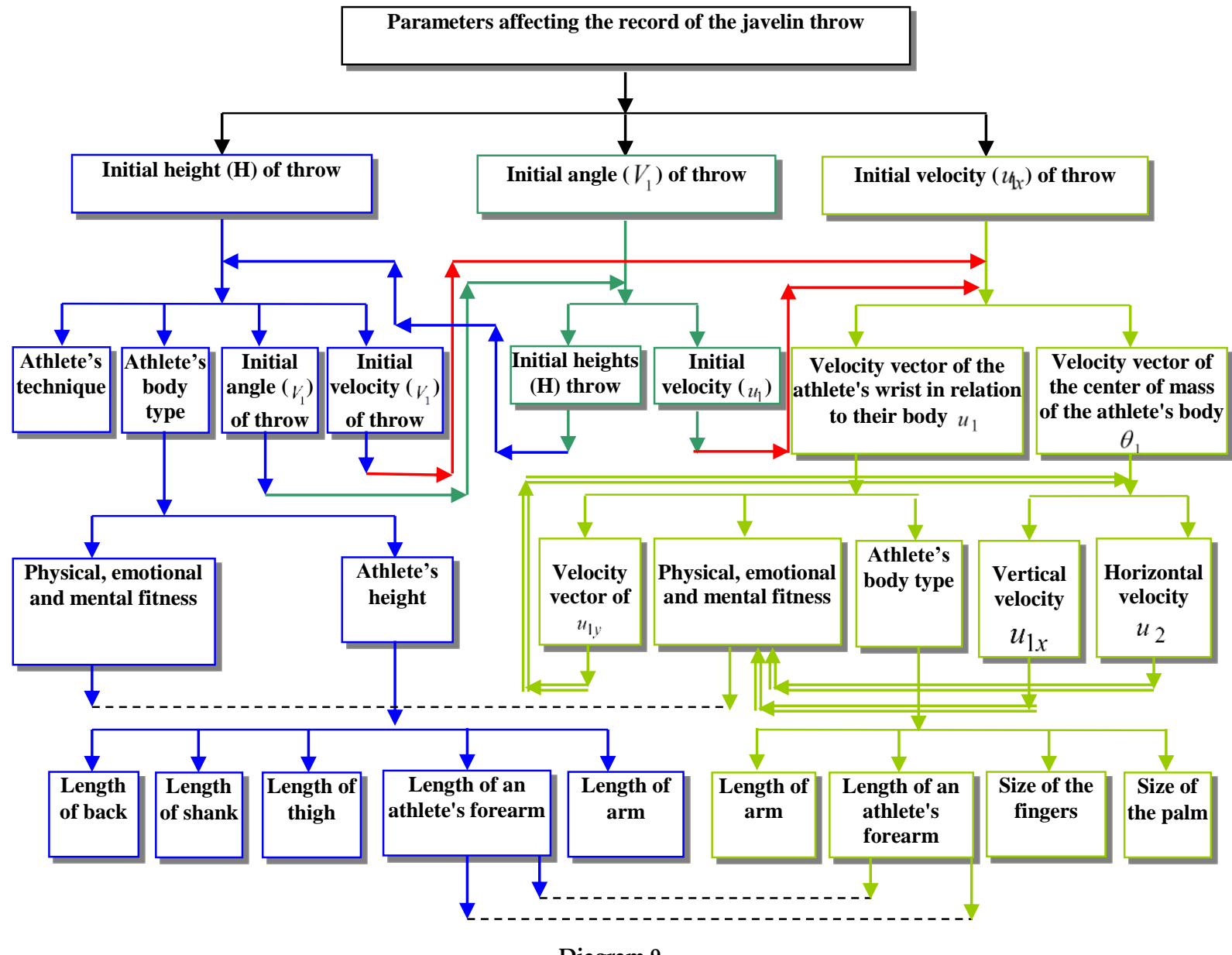

Diagram 9.

Diagram 9 shows that the parameters have intense relationship with throw record in a tree form, and how these components are related to each other is shown. However, partial changes in a parameter causes partial or total changes in several parameters, resulting in increase or decrease in some of the parameters and $\mathrm{r}$ simultaneously causes some changes in the record of a throw. Therefore, by correct management, precise calculation, recording and measuring the initial and existing values, this study aimed to find the optimal values of these three parameters and with the aid of these optimal values, the athlete's maximum record was achieved (Morriss et al., 1995; Kunz \& Kaufmann, 1983). The equations obtained and Diagrams 1 to 8 , represent an increase in the record, and in some cases, represent a decrease in the record. In these diagrams, it is observed that changes in the initial throw height towards increasing it, causes an increase in the record.
Changes in the throw angle towards increasing it, causes an increase and then a decrease in the record. Thus future studies are required to calculate the optimal value so as to determine the lunch angle.

Table 1 was obtained from the equations in this study and in this regard, a basic software program was prepared to investigate a projectile motion and determine the optimum modes, which have the ability to investigate and analyze the projectile motion, draw the relevant diagrams and display the motion. Therefore, future planning is required to prepare a more complete software program on the simulation of projectile motion involving several other parameters such as air resistance, wind, etc. In the light of these, the aim of this study is to achieve a comprehensive software program for a coach based on which athletes can be led during training in order 
to attain an efficiency of $100 \%$ in terms of record. Also this software program can be an appropriate criterion for the discovery of talents, in this sport event.

\section{References}

Bartlett RM, Best RJ. The biomechanics of javelin throwing: A review. Journal of sports sciences, 1988; 6(1): 1-38.

Bayat MR, Shadmehr B, Rajabi H, Khajavi N. Track and Field (Vol. 2): Jumping and Throwing Events. $9^{\text {th }}$ ed., Tehran: Organization of studying and compiling humanities books of universities (SAMT), 2015.

Best R, Bartlett R, Morriss C. A three-dimensional analysis of javelin throwing technique. Journal of sports sciences, 1993; 11(4): 315-328.

Bosen KO. Coaching Hammer Throwing Technique. Track and Field Quarterly Review, 1985; 85(1): 36-39.

Dai B, Leigh S, Li H, Mercer VS, Yu B. The relationships between technique variability and performance in discus throwing. Journal of Sports Sciences, 2013; 31(2): 219-228.

Dai B, Leigh S, Li H, Yu B. The relationships between technique variability and performance in elite discus throwers during competition. The 35th American Society of Biomechanics Annual Meeting, Long Beach, CA, 2011.
Kunz H, Kaufmann D. Cinematographical analysis of javelin throwing techniques of decathletes. British Journal of Sports Medicine, 1983; 17(3): 200-204.

David H, Robert R, Walker J. Fundamentals of physics. $11^{\text {th }}$ ed, John Wiley \& Sons Incorporated, 2018.

Leigh S, Gross MT, Li L, Yu B. The relationship between discus throwing performance and combinations of selected technical parameters. Sports Biomechanics, 2008; 7(2): 173-193.

Leigh S, Liu H, Yu B. Associations between javelin throwing technique and aerodynamic distance. ISBS-Conference Proceedings Archive, 2010.

Leigh S, Dapena J, Gross M, Li L, Myers J, Yu B. Associations between javelin throwing technique and upper extremity kinetics. The $31^{\text {st }}$ International Conference on Biomechanics in Sports, 2013.

Liu H, Leigh S, Yu B. Effects of movement sequence on the performance of javelin throwing. International Symposium on Biomechanics in Sports: Conference, 2010a; 28, 1.

Liu H, Leigh S, Yu B. Sequences of upper and lower extremity motions in javelin throwing. Journal of sports sciences, 2010b; 28(13): 1459-1467.

Morriss C, Bartlett R, Navarro E, Viitasalo J. The Biomechanics of Elite Javelin Throwing Technique. ISBS-Conference Proceedings Archive, 1995.

Robinson FC. Modern Technique of Track and Field. Provo, Utah, London: Brigham Young University, 2016. 